\title{
EFECTOS DEL ENTRENAMIENTO POR INTERVALOS VS ENTRENAMIENTO CONTINUO SOBRE LA CAPACIDAD AERÓBICA EN PACIENTES CON ENFERMEDAD DE LAS ARTERIAS CORONARIAS
}

\section{EFFECTS OF INTERVAL TRAINING VS CONTINUOUS TRAINING ON AEROBIC CAPACITY IN PATIENTS WITH CORONARY ARTERY DISEASE}

\author{
Bruno Bizzozero Peroni ${ }^{1}$ y Valentina Díaz Goñi ${ }^{1}$ \\ bbizzozero@isef.edu.uy \\ valediazgoni@gmail.com
}

\author{
${ }^{1}$ Instituto Superior de Educación Física, Universidad de la República, Uruguay \\ Envío: 2019-06-25 Reenviado: 2019-10-07, 2019-10-30 Aceptado: 2019-10-30
}

Publicado: 2019-11-26

Doi: https://doi.org/10.15517/pensarmov.v17i2.37766

\begin{abstract}
RESUMEN
El objetivo de esta revisión fue comparar la influencia del entrenamiento por intervalos de alta intensidad (HIIT) con el entrenamiento continuo de moderada intensidad (MICT) sobre la capacidad aeróbica y otras variables relevantes en pacientes con enfermedad de las arterias coronaria (EAC). Realizamos una revisión de revisiones sistemáticas y metaanálisis en PubMed hasta el 04 de junio de 2019. Se identificaron un total de 22 artículos, de los cuales 6 se seleccionaron finalmente para esta revisión. La calidad metodológica se evaluó utilizando la herramienta 'Assessment of Multiple Systematic Reviews 2' (AMSTAR2). Todos los estudios analizaron los efectos del HIIT y MICT en pacientes adultos (rango edad media: 52-76 años). La calidad general de los estudios incluidos fue moderada-alta (AMSTAR-2). EI HIIT presentó mayores mejorías sobre el consumo de oxígeno pico y la frecuencia cardíaca pico en comparación al MICT. Esta revisión agrega evidencia adicional que el HIIT presenta mejoras clínicamente significativas sobre la capacidad aeróbica en comparación al MICT. Son necesarios más estudios que permitan establecer conclusiones consistentes de los efectos del HIIT y MICT sobre otras variables relevantes.
\end{abstract}

Palabras claves: Capacidad aeróbica, enfermedad de las arterias coronarias, entrenamiento continuo de moderada intensidad, entrenamiento por intervalos de alta intensidad, rehabilitación cardíaca. 


\begin{abstract}
The aim of this review was to compare the influence of high-intensity interval training (HIIT) with moderate-intensity continuous training (MICT) on aerobic capacity and other relevant variables in patients with coronary artery disease (EAC). We conducted a review of systematic reviews and meta-analyzes in PubMed up to 4 June 2019. A total of 22 articles were identified, of which 6 were finally selected for this review. The methodological quality was evaluated using the 'Assessment of Multiple Systematic Reviews 2' (AMSTAR-2) tool. All the studies analyzed the effects of HIIT and MICT in adult patients (range average age: 52-76 years). The overall quality of the included studies was moderate-high (AMSTAR-2). HIIT showed greater improvements over peak oxygen consumption and peak heart rate compared to MICT. This review adds further evidence that HIIT presents clinically significant improvements in aerobic capacity compared to MICT. More studies are needed to establish consistent conclusions about the effects of HIIT and MICT on other relevant variables.
\end{abstract}

Keywords: aerobic capacity, coronary artery disease, moderate-intensity continuous training, high-intensity interval training, cardiac rehabilitation.

\title{
1. INTRODUCCIÓN
}

La enfermedad de las arterias coronarias (EAC) afecta a 17.5 millones de personas cada año (World Health Organization, 2016a), siendo la primera causa de muertes a nivel mundial con 7.4 millones de muertes en 2012 y un pronóstico de 9.2 millones de fallecimientos para el año 2030 (World Health Organization, 2011, 2016b). La rehabilitación cardíaca $(\mathrm{RC})$ es el tratamiento y prevención secundaria de enfermedades cardíacas (Montalescot et al., 2013). La RC mejora el pronóstico de la enfermedad, recomendándose la participación de todos los pacientes con EAC (Woodruffe et al., 2015). Es recomendable la inclusión de un programa de ejercicio físico (PEF) dentro de la RC como factor principal para el éxito de esta y la prevención en la recurrencia de eventos cardíacos (Piepoli et al., 2010). Las actuales cifras de mortalidad por EAC junto a su pronóstico de aumento para los próximos años, suponen un problema socio-sanitario de primer orden mundial (World Health Organization, 2016b). Por ello, la RC con un PEF resultan cruciales para reducir estas cifras y mejorar la salud de los pacientes (Montalescot et al., 2013; Woodruffe et al., 2015). De hecho, la RC que incluye un PEF se asocia con mejoras en funciones cardiovasculares y mortalidad cardiovascular en pacientes con EAC (Anderson et al., 2016). Estas mejoras están mediadas, entre otros elementos, a través del incremento de la capacidad aeróbica inducido por el PEF (Valkeinen, Aaltonen y Kujala, 2010). La capacidad aeróbica, determinada generalmente a través del consumo máximo de oxígeno $\left(\mathrm{VO}_{2 m a ́ x}\right)$ o el consumo 
de oxígeno pico $\left(\mathrm{VO}_{2 \text { pico }}\right)$, es un predictor importante en el pronóstico y mortalidad en esta enfermedad (Kodama et al., 2009).

El entrenamiento aeróbico continuo de moderada intensidad (MICT) ha sido el PEF tradicional en la $\mathrm{RC}$, con mejoras sobre el $\mathrm{VO}_{2 \text { pico }}$ en pacientes con EAC (Piepoli et al., 2010). Pero entrenar a intensidades más altas conduce a mayores mejorías sobre el $\mathrm{VO}_{2 \text { pico }}$ (Moholdt, Madssen, Rognmo y Aamot, 2014). Para prolongar el tiempo del entrenamiento a intensidades altas, surge el entrenamiento por intervalos (Mezzani et al., 2013). El entrenamiento por intervalos de alta intensidad (HIIT) consiste en períodos cortos (0.5-4 minutos) de ejercicio físico a alta intensidad (85-95\% $\mathrm{VO}_{2 \text { pico }}$ ) de forma intermitente con períodos de recuperación activos $\left(50-70 \% \mathrm{VO}_{2 \text { pico }}\right.$ ) o pasivos (Ballesta García, Rubio Arias, Ramos Campo, Martínez González-Moro y Carrasco Poyatos, 2019). EI HIIT se introdujo y adaptó en la RC para pacientes con EAC hace unos 15 años, reportando mayores mejorías sobre la capacidad aeróbica en comparación al MICT (Rognmo, Hetland, Helgerud, Hoff y Slørdahl, 2004). Sin embargo, los resultados de estudios individuales son contradictorios y los estudios de revisiones sistemáticas no se han comparado (Pattyn, Beulque y Cornelissen, 2018). Por lo tanto, el objetivo del presente estudio es analizar la eficacia del HIIT en comparación al MICT sobre la capacidad aeróbica y otras variables relevantes de la $\mathrm{RC}$ en pacientes con EAC.

\section{MÉTODO}

\subsection{Estrategia de búsqueda}

Se realizó una búsqueda electrónica de revisiones hasta el 04 de junio de 2019 en la base de datos PubMed. Se incluyeron revisiones sistemáticas y/o meta-análisis que examinaran y compararan efectos del HIIT y MICT sobre la capacidad aeróbica como variable principal de la $\mathrm{RC}$ en pacientes con EAC.

Al realizar la búsqueda en PubMed se utilizaron los términos MeSH (Medical Subject Heading) para definir la patología cardíaca y la intervención mediante ejercicio físico (ver tabla 1). La combinación fue la siguiente: 'coronary artery disease' [MeSH] AND 'exercise' [MeSH] OR 'exercise therapy' [MeSH] OR 'cardiac rehabilitation' [MeSH] OR 'high-intensity interval training' [MeSH]. Además, se seleccionaron como criterios para la búsqueda: estudios de revisiones sistemáticas y/o meta-análisis, publicados en inglés o español, a texto completo, y en población humana. 


\subsection{Criterios de inclusión/exclusión}

Para ser seleccionados en esta revisión, los artículos debían cumplir los siguientes criterios de inclusión: (1) estudios que analicen la influencia del HIIT en comparación al MICT sobre la capacidad aeróbica como variable principal de la RC en pacientes con EAC; (2) en inglés o español a texto completo, publicados en la base de datos seleccionada; y, (3) en modalidad de revisión sistemática y/o meta-análisis.

Además, los criterios de exclusión fueron los siguientes: (1) investigaciones sobre pacientes cardíacos que no incluyan EAC; (2) revisiones que no expresen resultados específicos sobre la capacidad aeróbica; (3) estudios que no comparen los efectos del HIIT y MICT; (4) revisiones que sus resultados sean la combinación de EAC con otra patología no cardíaca; $y,(5)$ artículos que expresen resultados en base a estudios en animales.

\subsection{Identificación de estudios}

Siguiendo los procedimientos de la estrategia de búsqueda en la base de datos PubMed, se identificaron 22 artículos. La figura 1 muestra gráficamente el flujo del proceso de búsqueda según la declaración 'Preferred Reporting Items for Systematic Reviews and Meta-Analyses' (PRISMA) (Moher, Liberati, Tetzlaff, Altman y Group, 2009). En base a los criterios de inclusión/exclusión, dos revisores (BBP y VDG) realizaron el siguiente procedimiento de selección: (1) fase de cribado aplicada a título y resumen; (2) búsqueda de texto completo y evaluación de elegibilidad de los artículos seleccionados después del paso anterior. Se buscaron manualmente las listas de referencias de los artículos incluidos para identificar otros estudios apropiados. Finalmente, un total de 6 artículos cumplieron con los criterios de inclusión/exclusión.

\subsection{Extracción de datos}

Dos revisores (BBP y VDG) recopilaron los datos de las revisiones sistemáticas y/o metaanálisis que incluyeron: bases de datos utilizadas, años de búsqueda de artículos, tipo y cantidad de artículos, criterios de inclusión, edad de los pacientes, descripción de las características del entrenamiento, resultados de los efectos de los entrenamientos sobre la capacidad aeróbica y variables secundarias, y resultados de los efectos de las características del entrenamiento sobre la capacidad aeróbica. 


\subsection{Evaluación de la calidad de los estudios}

La calidad metodológica se evaluó utilizando la herramienta 'Assessment of Multiple Systematic Reviews 2' (AMSTAR-2), instrumento validado para la evaluación crítica de revisiones sistemáticas que incluyan ensayos aleatorizados y no aleatorizados (Shea et al., 2017a). AMSTAR-2 es un cuestionario que contiene 16 dominios con opciones de respuesta: "si" (resultado es positivo), "si parcial" (adherencia parcial al estándar), o "no" no se cumplió el estándar) (Shea et al., 2017a). 7 dominios son considerados críticos (dado que pueden afectar sustancialmente la validez de una revisión y sus conclusiones, y 9 dominios son considerados no críticos (ver tabla 2). De las debilidades en estos dominios surgen cuatro niveles de confianza: alta (ninguna debilidad crítica y hasta una no crítica), moderada (ninguna debilidad crítica y más de una debilidad crítica), baja (hasta una debilidad no crítica, con o sin debilidades no críticas) y críticamente baja (más de una debilidad crítica, con o sin debilidades no críticas) (Shea et al., 2017a). Los autores realizaron la valoración de confianza de los estudios utilizando la lista de verificación en línea AMSTAR-2 (Shea et al., 2017b). Cada uno de los estudios fue analizado por dos revisores (BBP y VDG), siendo las evaluaciones discutidas y acordadas por ambos. La tabla 2 resume la evaluación de calidad de las revisiones sistemáticas y/o meta-análisis incluidos.

\section{RESULTADOS}

\subsection{Características de los estudios incluidos}

Once estudios fueron descartados al aplicar criterios de inclusión/exclusión al título y resumen. Del total de 11 estudios de texto completo evaluados para elegibilidad, se excluyeron 5 al aplicar criterios de inclusión/exclusión (Ballesta García et al., 2019; Clark, Hartling, Vandermeer y McAlister, 2005; Cornish, Broadbent y Cheema, 2011; Guiraud et al., 2012; Kraal, Vromen, Spee, Kemps y Peek, 2017). En total se incluyeron 6 estudios, 5 identificados en la búsqueda de la base de datos PubMed (Elliott, Rajopadhyaya, Bentley, Beltrame y Aromataris, 2015; Gomes-Neto et al., 2017; Liou, Ho, Fildes y Ooi, 2016; Pattyn et al., 2018; Pattyn, Coeckelberghs, Buys, Cornelissen y Vanhees, 2014) y 1 identificado en la búsqueda manual (Hannan et al., 2018) (Fig. 1). 


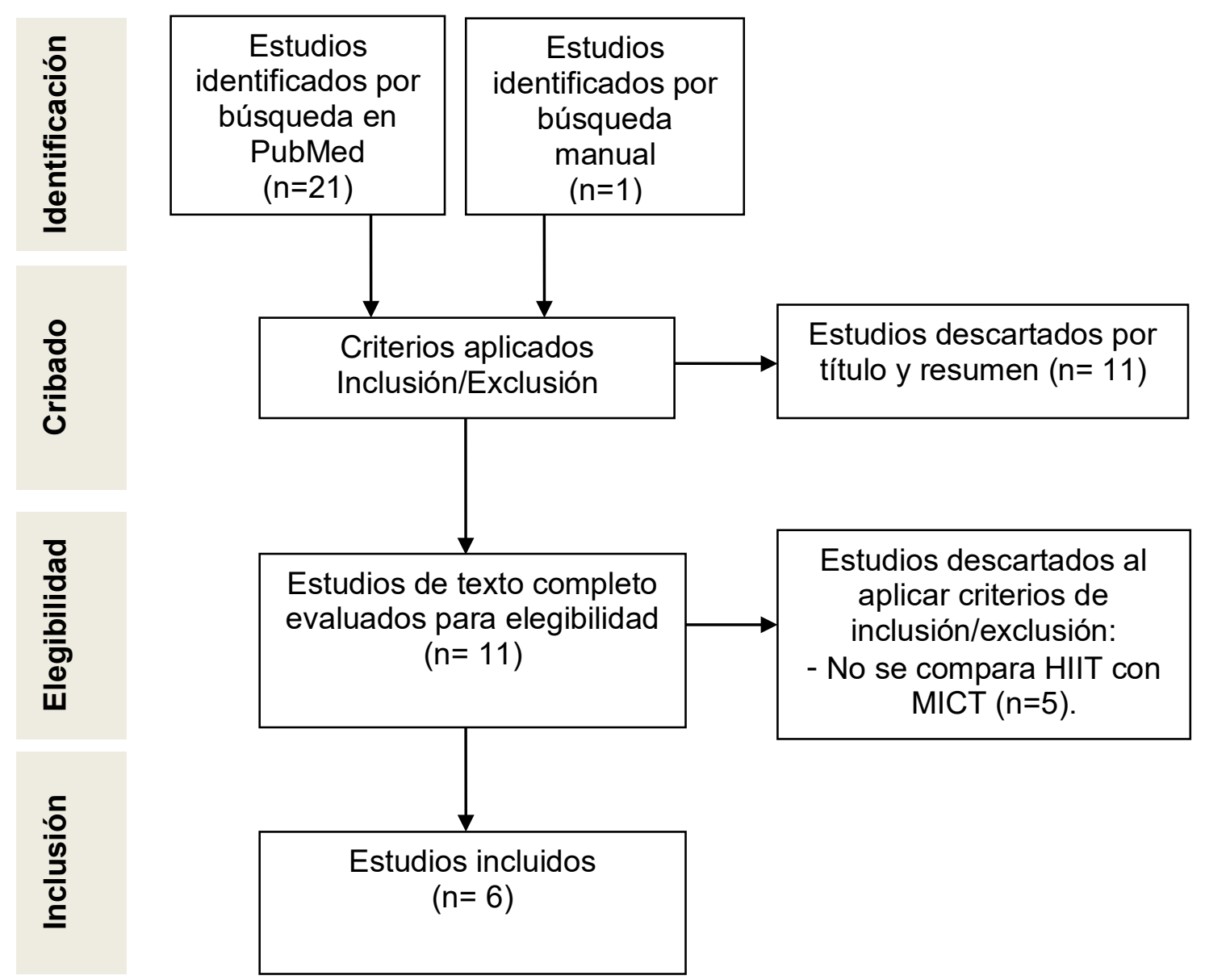

Figura 1. Diagrama de flujo según PRISMA para la selección de revisiones sistemáticas y meta-análisis.

Un resumen de los estudios incluidos se presenta en la tabla 1. Dos estudios son metaanálisis y 4 contienen revisiones sistemáticas y meta-análisis. Se analizaron 64 artículos en los 6 estudios incluidos, de las cuales se encontraron un total de 24 intervenciones diferentes (ver Apéndice 1) con 1250 pacientes adultos (rango edad media: 52-76 años). Los resultados de la capacidad aeróbica se determinaron a través del $\mathrm{VO}_{2 \text { pico }}$ (Elliott et al., 2015; Gomes-Neto et al., 2017; Hannan et al., 2018; Liou et al., 2016; Pattyn et al., 2018, 2014). Las variables secundarias que se analizaron en los estudios incluidos fueron: calidad de vida (Gomes-Neto et al., 2017; Pattyn et al., 2014), dilatación mediada por flujo, fracción de eyección del ventrículo izquierdo (Pattyn et al., 2018), frecuencia cardíaca de reposo (Liou et al., 2016; Pattyn et al., 2018), frecuencia cardíaca pico (Pattyn et al., 2018), nivel de lípidos (Elliott et al., 2015; Pattyn et al., 2018), nivel de glucosa (Pattyn et al., 2018), peso corporal (Liou et al., 2016; Pattyn et al., 2018, 2014), presión arterial (Elliott et al., 2015; Pattyn et al., 2018), pendiente de eficiencia de consumo de oxígeno (Pattyn et al., 2018), pendiente de eficiencia ventilatoria, primer umbral ventilatorio (Pattyn et al., 2018, 2014), y pulso de oxígeno (Pattyn et al., 2018). 
Tabla 1.

Características de los estudios incluidos.

\begin{tabular}{|c|c|c|c|c|c|c|}
\hline Estudios & $\begin{array}{l}\text { Tipo de } \\
\text { estudio }\end{array}$ & Bases de datos utilizadas & $\begin{array}{c}\text { Búsqueda } \\
\text { hasta }(\mathrm{m} / \mathrm{a})\end{array}$ & Criterios inclusión & $\begin{array}{l}\text { Artículos } \\
\text { incluidos (n) }\end{array}$ & $\begin{array}{l}\text { Pacientes } \\
\text { (edad) }\end{array}$ \\
\hline $\begin{array}{l}\text { Gomes Neto } \\
\text { et al. }(\underline{2017})^{*}\end{array}$ & $\begin{array}{r}\text { RS y } \\
\text { MA }\end{array}$ & $\begin{array}{l}\text { Cochrane Library, Medline, } \\
\text { LILACS, PEDro, SciELO }\end{array}$ & $11 / 16$ & $\begin{array}{l}\text { (1) RCTs que comparen efectos del HITT y MICT sobre CA o calidad de } \\
\text { vida; y (2) en pacientes con EAC }\end{array}$ & 11 & $55-65$ \\
\hline $\begin{array}{l}\text { Hannan et al. } \\
\qquad(\underline{2018})^{*}\end{array}$ & $\begin{array}{r}\text { RS y } \\
\text { MA }\end{array}$ & $\begin{array}{l}\text { CINAHL, Embase, Medline, } \\
\text { SPORTDiscus, WOS }\end{array}$ & $7 / 17$ & $\begin{array}{l}\text { (1) RCTs que comparen efectos pre-post intervención del HIIT y MICT } \\
\text { sobre CA como variable principal; (2) en pacientes con EAC; (3) PEF con } \\
\text { un mínimo de } 4 \text { semanas; y (4) publicados en revistas revisadas por } \\
\text { pares a texto completo }\end{array}$ & 17 & $52-76$ \\
\hline $\begin{array}{l}\text { Pattyn et al. } \\
(\underline{\underline{2018}})^{*}\end{array}$ & $\begin{array}{r}\text { RS y } \\
\text { MA }\end{array}$ & PubMed, SPORTDiscus & $3 / 17$ & $\begin{array}{c}\text { (1) RCTs o ECA que comparen efectos del HIIT y MICT; (2) PEF con un } \\
\text { mínimo de } 4 \text { semanas; (3) informen la media y DE de la CA y variables } \\
\text { secundarias pre-post intervención; (4) pacientes con EAC o IC; y (5) } \\
\text { publicados en revistas revisadas por pares }\end{array}$ & 11 & $55-68$ \\
\hline $\begin{array}{l}\text { Elliott et al. } \\
(\underline{\underline{2015}})^{*}\end{array}$ & MA & $\begin{array}{l}\text { ANZCTR, CINAHL, } \\
\text { clinicaltrials.gov, Embase, } \\
\text { PubMed, TROVE }\end{array}$ & $12 / 13$ & $\begin{array}{l}\text { (1) RCTs que comparen efectos del HIIT y MICT en pacientes con EAC y } \\
\text { ausencia de IC; (2) PEF con un mínimo de } 4 \text { semanas; y (3) reporten } \\
\text { resultados sobre CA }\end{array}$ & 6 & $55-68$ \\
\hline $\begin{array}{l}\text { Liou et al. } \\
(\underline{\underline{2016}})^{*}\end{array}$ & MA & Embase, Medline & $5 / 15$ & $\begin{array}{c}\text { (1) RCTs que comparen HIIT y MICT en las variables de interés; (2) PEF } \\
\text { con un mínimo de } 4 \text { semanas; (3) pacientes con EAC y FEVI reducida o } \\
\text { preservada; y (4) reporten datos en bruto con el } 95 \% \text { del intervalo de } \\
\text { confianza }\end{array}$ & 10 & $55-76$ \\
\hline $\begin{array}{l}\text { Pattyn et al. } \\
(\underline{\underline{2014}})^{*}\end{array}$ & $\begin{array}{r}\text { RS y } \\
\text { MA }\end{array}$ & PubMed & $5 / 13$ & $\begin{array}{l}\text { (1) Estudios de intervención aleatorizada publicados en revistas } \\
\text { revisadas por pares; (2) comparen HIIT y MICT (supervisados); (3) PEF } \\
\text { con un mínimo de } 4 \text { semanas; (4) pacientes con EAC y FEVI reducida o } \\
\text { preservada; y (5) informen la media y DE de la CA pre-post intervención }\end{array}$ & 9 & $55-68$ \\
\hline
\end{tabular}


La tabla 2 indica los niveles de confianza AMSTAR-2. La calidad general de los estudios incluidos fue moderada-alta. Del total de 6 estudios incluidos, 3 estudios presentaron nivel de confianza alto (Gomes-Neto et al., 2017; Hannan et al., 2018; Pattyn et al., 2018), 2 presentaron nivel de confianza moderado (Elliott et al., 2015; Liou et al., 2016), y 1 presentó nivel de confianza bajo (Pattyn et al., 2014).

\section{Tabla 2.}

Evaluación de la calidad metodológica (AMSTAR-2) de revisiones sistemáticas y meta-análisis incluidos.

\section{Dominios / Ítems}

\begin{tabular}{|c|c|c|c|c|c|c|c|c|c|c|c|c|c|c|c|c|c|}
\hline Estudios & 1 & 2 & 3 & 4 & 5 & 6 & 7 & 8 & 9 & 10 & 11 & 12 & 13 & 14 & 15 & 16 & Confianza \\
\hline Gomes-Neto et al. (2017) & $\mathrm{Si}$ & S/P & $\mathrm{Si}$ & S/P & $\mathrm{Si}$ & Si & S/P & Si & Si & No & Si & $\mathrm{Si}$ & Si & $\mathrm{Si}$ & S/P & $\mathrm{Si}$ & Alta \\
\hline Hannan et al. (2018) & $\mathrm{Si}$ & Si & $\mathrm{Si}$ & Si & $\mathrm{Si}$ & $\mathrm{Si}$ & $\mathbf{S} / \mathbf{P}$ & $\mathrm{Si}$ & Si & No & Si & $\mathrm{Si}$ & Si & $\mathrm{Si}$ & Si & Si & Alta \\
\hline Pattyn et al. (2018) & Si & $\mathbf{S} / \mathbf{P}$ & $\mathrm{Si}$ & S/P & $\mathrm{Si}$ & Si & Si & $\mathrm{Si}$ & Si & No & Si & $\mathrm{Si}$ & Si & $\mathrm{Si}$ & Si & $\mathrm{Si}$ & Alta \\
\hline Liou et al. (2016) & $\mathrm{Si}$ & S/P & $\mathrm{Si}$ & S/P & $\mathrm{Si}$ & $\mathrm{Si}$ & Si & $\mathrm{Si}$ & Si & No & Si & $\mathrm{Si}$ & Si & $\mathrm{Si}$ & Si & No & Moderada \\
\hline Pattyn et al. (2014) & $\mathrm{Si}$ & S/P & $\mathrm{Si}$ & No & No & $\mathrm{Si}$ & S/P & $\mathrm{Si}$ & Si & No & Si & $\mathrm{Si}$ & Si & $\mathrm{Si}$ & Si & $\mathrm{Si}$ & Baja \\
\hline
\end{tabular}

AMSTAR-2 contiene 7 dominios críticos (ítems 2, 4, 7, 9, 11, 13, 15) y 9 dominios no críticos que pueden ser calificados como "si", "si parcial" (S/P), "no", o "no aplica" (N/A). Shea et al. (2017a) establecen las preguntas de los dominios/ítems de AMSTAR-2.

\subsection{Características de las intervenciones en los estudios incluidos}

Para el HIIT y MICT, la duración osciló entre 4-48 semanas, la frecuencia osciló entre 2-5 sesiones semanales, y los tipos de ejercicio físico más utilizados fueron en cinta de correr y cicloergómetro (Elliott et al., 2015; Gomes-Neto et al., 2017; Hannan et al., 2018; Liou et al., 2016; Pattyn et al., 2018, 2014). Para el HIIT, el rango de intensidades más utilizadas en los intervalos de alta intensidad osciló entre $73-95 \% \mathrm{FC}_{\text {máx }}, 80-95 \% \mathrm{FC}_{\text {pico }}-\mathrm{FC}_{\text {res }}-\mathrm{VO}_{2 \text { pico, }}$ 80$110 \%$ PPO (Peak Power Output, o potencia máxima generada), $80-90 \% \mathrm{VO}_{2 \text { máx }}$ y $85-95 \%$ $\mathrm{VO}_{2 \text { res }}$ (Elliott et al., 2015; Gomes-Neto et al., 2017; Hannan et al., 2018; Liou et al., 2016; Pattyn et al., 2018, 2014). El período de recuperación fue activo y las intensidades más utilizadas oscilaron entre $60-70 \% \mathrm{FC}_{\text {máx }}, 40-70 \% \mathrm{FC}_{\text {res }}$ y $50-60 \% \mathrm{VO}_{2 \text { pico }}$ (Hannan et al., 2018; Pattyn et al., 2018, 2014). El volumen por sesión del HIIT se detalló a través de los intervalos de alta intensidad de corta duración (8-15 x 1-2 minutos y 30-40 x 0.5-1 minuto) y larga duración (4-7 x 3-4 minutos) (Elliott et al., 2015; Hannan et al., 2018; Pattyn et al., 
2018, 2014). Para el MICT, el rango de intensidades más utilizadas osciló entre $60-83 \%$ $\mathrm{FC}_{\text {máx }}, 60-80 \% \mathrm{FC}_{\text {pico, }}, 50-85 \% \mathrm{FC}_{\text {res }}, 51-65 \% \mathrm{PPO}$ y $50-78 \% \mathrm{VO}_{2 \text { pico }}-\mathrm{VO}_{2 \text { máx }}-\mathrm{VO}_{2 \text { res }}$ (Elliott et al., 2015; Gomes-Neto et al., 2017; Hannan et al., 2018; Liou et al., 2016; Pattyn et al., $\underline{2018}, \underline{2014}$ ). El volumen por sesión del MICT osciló entre 15-50 minutos (Elliott et al., 2015; Gomes-Neto et al., 2017; Hannan et al., 2018; Pattyn et al., 2018, 2014). Tres estudios no establecieron el tipo e intensidad del período de recuperación del HIIT (Elliott et al., 2015; Gomes-Neto et al., 2017; Liou et al., 2016), 2 estudios no determinaron el volumen por sesión del HIIT (Gomes-Neto et al., 2017; Liou et al., 2016), 1 estudio no especificó el tipo de ejercicio utilizado en el HIIT y MICT (Gomes-Neto et al., 2017), y 1 estudio no detalló el volumen por sesión del MICT (Liou et al., 2016), Todas estas características vienen incluidas en las tablas 3 y 4 . 


\section{Tabla 3.}

Características del HIIT en las intervenciones de los estudios incluidos.

\begin{tabular}{|c|c|c|c|c|c|c|}
\hline \multirow[t]{2}{*}{ Estudios } & \multicolumn{5}{|c|}{ Características del HIIT } & \multirow[b]{2}{*}{$\begin{array}{l}\text { Volumen EF por sesión } \\
\quad \text { (rango int } x \text { min) }\end{array}$} \\
\hline & $\begin{array}{c}\text { Frecuencia } \\
\text { (rango ss) }\end{array}$ & $\begin{array}{l}\text { Duración programa } \\
\quad \text { (rango sem) }\end{array}$ & Intervalos & Intensidad & Tipo & \\
\hline \multirow[t]{2}{*}{$\begin{array}{l}\text { Gomes Neto } \\
\text { et al. }(\underline{2017})\end{array}$} & $2-5$ & $4-16$ & Al & $\begin{array}{l}73-93 \% \mathrm{FC}_{\text {máx }}, 85-95 \% \mathrm{FC}_{\text {pico }}, 80-90 \% \\
\mathrm{FC}_{\text {res }}, 80-90 \% \mathrm{VO}_{2 \text { pico }}, 85-95 \% \mathrm{VO}_{2 \text { res }}\end{array}$ & nse & nse \\
\hline & & & PR & nse & nse & nse \\
\hline $\begin{array}{l}\text { Hannan et al. } \\
\qquad(\underline{2018})\end{array}$ & $2-5$ & $4-48$ & $\mathrm{Al}$ & $\begin{array}{c}85-95 \% \mathrm{FC}_{\text {máx }}, 90-95 \% \mathrm{FC}_{\text {pico }}, 80-95 \% \\
\mathrm{FC}_{\text {res }}, 80-104 \% \mathrm{PPO}, \mathrm{pcr}, 70-95 \% \text { ua, } 80- \\
95 \% \mathrm{VO}_{2 \text { pico }}\end{array}$ & $\begin{array}{l}\text { cinta de correr, ciclo-ergómetro, } \\
\text { brazo-ergómetro, escaladora }\end{array}$ & $\begin{array}{l}\text { corto: } 8-10 \text { x 1-2 } \\
\text { largo: } 4-7 \text { x 3-4 }\end{array}$ \\
\hline \multirow{3}{*}{$\begin{array}{l}\text { Pattyn et al. } \\
\qquad \underline{(2018)}\end{array}$} & \multirow{3}{*}{$2-5$} & \multirow{3}{*}{$4-16$} & PR & $\begin{array}{c}60-70 \% \mathrm{FC}_{\text {máx }}, 50-70 \% \mathrm{FC}_{\text {res }}, 10 \% \mathrm{PPO} \\
\text { ua, } 50-60 \% \mathrm{VO}_{2 \text { pico }}\end{array}$ & Activo & $\begin{array}{l}\text { corto: } 7-10 \times 1-2 \\
\text { largo: } 4-7 \times 3\end{array}$ \\
\hline & & & Al & $\begin{array}{c}85-95 \% \mathrm{FC}_{\text {pico, }}, 80-95 \% \mathrm{FC}_{\text {res }}, 89-104 \% \\
\text { PPO, } 80-90 \% \mathrm{VO}_{2 \text { pico }}\end{array}$ & $\begin{array}{l}\text { cinta de correr, ciclo-ergómetro, } \\
\text { brazo-ergómetro, escaladora }\end{array}$ & $\begin{array}{l}\text { corto: } 8-15 \text { x 1-2 } \\
\text { largo: } 4-7 \text { x 3-4 }\end{array}$ \\
\hline & & & PR & $\begin{array}{c}50-70 \% \mathrm{FC}_{\text {pico }}, 35-70 \% \mathrm{FC}_{\text {res }}, 10 \% \mathrm{PPO} \\
\text { puv, } 50-60 \% \mathrm{VO}_{2 \text { pico }}\end{array}$ & Activo & $\begin{array}{c}\text { corto: } 8-15 \times 1-2 \\
\text { largo: } 4-7 \times 3\end{array}$ \\
\hline $\begin{array}{l}\text { Elliott et al. } \\
\qquad \underline{(2015})\end{array}$ & $2-5$ & $4-16$ & Al & $\begin{array}{c}90 \% \text { FCpico, } 80-90 \% \text { FCres, } 80-104 \% \\
\text { PPO, 80-90\% VO2pico, 90\% VO2res }\end{array}$ & $\begin{array}{c}\text { cinta de correr, ciclo-ergómetro, } \\
\text { brazo-ergómetro }\end{array}$ & $\begin{array}{l}\text { corto: } 8-10 \times 1-2 \\
\text { largo: } 4 \text { × } 4\end{array}$ \\
\hline
\end{tabular}




\section{Continuación Tabla 3.}

\begin{tabular}{|c|c|c|c|c|c|c|}
\hline \multirow[t]{2}{*}{ Estudios } & \multicolumn{5}{|c|}{ Características del HIIT } & \multirow[b]{2}{*}{$\begin{array}{l}\text { Volumen EF por sesión } \\
\text { (rango int } x \text { min) }\end{array}$} \\
\hline & $\begin{array}{l}\text { Frecuencia } \\
\text { (rango ss) }\end{array}$ & $\begin{array}{l}\text { Duración programa } \\
\text { (rango sem) }\end{array}$ & Intervalos & Intensidad & Tipo & \\
\hline $\begin{array}{l}\text { Elliott et al. } \\
\qquad \underline{\underline{2015}})\end{array}$ & & & PR & nse & nse & $\begin{array}{c}\text { corto: } 7-10 \times 1-2 \\
\text { largo: } 4 \times 3\end{array}$ \\
\hline \multirow[t]{2}{*}{$\begin{array}{l}\text { Liou et al. } \\
\qquad \underline{\underline{2016}})\end{array}$} & $3-5$ & $4-16$ & $\mathrm{Al}$ & $\begin{array}{l}\text { 80-95\% FCpico, } 80-104 \% \text { PPO, } 90 \% \\
\text { VO2res }\end{array}$ & Ciclo-ergómetro & nse \\
\hline & & & PR & nse & nse & nse \\
\hline \multirow[t]{2}{*}{$\begin{array}{l}\text { Pattyn et al. } \\
\quad(\underline{2014})\end{array}$} & $2-5$ & $4-16$ & $\mathrm{Al}$ & $\begin{array}{l}\text { 85-95\% FCmáx, 90\% FCres / VO2res, pcr, } \\
\text { 80-110\% PPO, 80-90\% VO2máx }\end{array}$ & $\begin{array}{l}\text { cinta de correr, ciclo-ergómetro, } \\
\text { brazo-ergómetro, escaladora }\end{array}$ & $\begin{array}{l}\text { corto: } 30-40 \text { x 0.5-1 } \\
\text { corto: } 8-10 \text { x 1-2 } \\
\text { largo: } 4-7 \text { × 3-4 }\end{array}$ \\
\hline & & & PR & $\begin{array}{l}\text { 70\% FCmáx, 40\% FCres / VO2res, } 10 \% \\
\text { PPO, puv, 50-60\% VO2pico }\end{array}$ & Activo & $\begin{array}{c}\text { corto: } 30-40 \text { x 0.5-1 } \\
\begin{array}{c}\text { corto: } 7-10 \times 1-2 \\
\text { largo: } 4-7 \times 3\end{array}\end{array}$ \\
\hline
\end{tabular}




\section{Tabla 4.}

Características del MICT en las intervenciones de los estudios incluidos.

\begin{tabular}{|c|c|c|c|c|c|}
\hline \multirow[t]{2}{*}{ Estudios } & \multicolumn{4}{|c|}{ Características del MICT } & \multirow[b]{2}{*}{$\begin{array}{l}\text { Volumen EF por } \\
\text { sesión (rango min) }\end{array}$} \\
\hline & $\begin{array}{c}\text { Frecuencia } \\
\text { (rango ss) }\end{array}$ & $\begin{array}{l}\text { Duración programa } \\
\quad \text { (rango sem) }\end{array}$ & Intensidad & Tipo & \\
\hline $\begin{array}{l}\text { Gomes Neto et } \\
\quad \text { al. }(\underline{2017})\end{array}$ & $2-5$ & $4-16$ & $\begin{array}{c}63-83 \% \mathrm{FC}_{\text {máx }}, \\
\qquad 0-80 \% \mathrm{FC}_{\text {pico }}, 60-80 \% \mathrm{FC}_{\text {res }}, 50-60 \% \\
\mathrm{VO}_{2 \text { pico, }}, 65 \% \mathrm{VO}_{2 \text { res }}\end{array}$ & nse & $15-50$ \\
\hline $\begin{array}{l}\text { Hannan et al. } \\
\qquad(\underline{2018})\end{array}$ & $2-5$ & $4-48$ & $\begin{array}{l}70-75 \% \mathrm{FC}_{\text {máx }}, 70-80 \% \mathrm{FC}_{\text {pico, }}, 60-85 \% \mathrm{FC}_{\text {res }}, 51-65 \% \\
\mathrm{PPO}, 60 \% \text { ua, } 50-78 \% \mathrm{VO}_{2 \text { pico }}, 60 \% \mathrm{VO}_{\text {res }}\end{array}$ & $\begin{array}{l}\text { cinta de correr, ciclo-ergómetro, } \\
\text { brazo-ergómetro, escaladora }\end{array}$ & $20-50$ \\
\hline $\begin{array}{l}\text { Pattyn et al. } \\
\qquad(\underline{2018})\end{array}$ & $2-5$ & $4-16$ & $\begin{array}{c}60-75 \% \mathrm{FC}_{\text {pico }}, 50-80 \% \mathrm{FC}_{\text {res }}, 51-65 \% \mathrm{PPO} \text {, puv, } 50-60 \% \\
\mathrm{VO}_{2 \text { pico }}\end{array}$ & $\begin{array}{l}\text { cinta de correr, ciclo-ergómetro, } \\
\text { brazo-ergómetro, escaladora }\end{array}$ & $40-50$ \\
\hline $\begin{array}{l}\text { Elliott et al. } \\
\qquad \underline{(2015})\end{array}$ & $2-5$ & $4-16$ & $\begin{array}{c}70 \% \mathrm{FC}_{\text {máx }}, 60-80 \% \mathrm{FC}_{\text {res }}, 51-65 \% \mathrm{PPO}, 50-60 \% \mathrm{VO}_{2 \text { pico }} \\
65 \% \mathrm{VO}_{\text {res }}\end{array}$ & $\begin{array}{l}\text { cinta de correr, ciclo-ergómetro, } \\
\text { brazo-ergómetro, escaladora }\end{array}$ & $30-50$ \\
\hline $\begin{array}{l}\text { Liou et al. } \\
\qquad(\underline{2016})\end{array}$ & $2-5$ & $4-16$ & $\begin{array}{l}63-83 \% \mathrm{FC}_{\text {máx }}, 70-80 \% \mathrm{FC}_{\text {pico, }}, 60-80 \% \mathrm{FC}_{\text {res }}, 51-65 \% \\
\text { PPO, } 50-60 \% \mathrm{VO}_{2 \text { pico }}, 65 \% \mathrm{VO}_{2 \text { res }}\end{array}$ & Ciclo-ergómetro & nse \\
\hline $\begin{array}{l}\text { Pattyn et al. } \\
\qquad \underline{(2014)}\end{array}$ & $2-5$ & $4-12$ & $70 \% \mathrm{FC}_{\text {máx }}$ uav, $51-65 \% \mathrm{PPO}, 50-60 \% \mathrm{VO}_{2 \text { máx }}$ & $\begin{array}{l}\text { cinta de correr, ciclo-ergómetro, } \\
\text { brazo-ergómetro, escaladora }\end{array}$ & $30-50$ \\
\hline
\end{tabular}

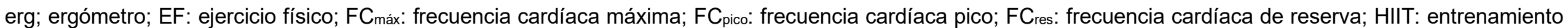
por intervalos de alta intensidad; min: minutos; nse: no se especifica; PPO: potencia máxima generada; sem: semanas; ss: sesiones semanales; ua: umbral de angina; $\mathrm{VO}_{2 m a ́ x:}$ consumo máximo de oxígeno; $\mathrm{VO}_{2 p i c o}$ : consumo de oxígeno pico; $\mathrm{VO}_{2 \text { res: }}$ consumo de oxígeno de reserva. 


\subsection{Efectos del HIIT vs MICT sobre la capacidad aeróbica}

El HIIT se asoció con mejoras significativas sobre el $\mathrm{VO}_{2 p i c o}$ en comparación al MICT (rango medio: +1.15 a $+1.78 \mathrm{~mL} / \mathrm{kg} / \mathrm{min}$ ) (Elliott et al., 2015; Gomes-Neto et al., 2017; Hannan et al., 2018; Liou et al., 2016; Pattyn et al., 2018, 2014). En la tabla 5 se presenta un resumen de estos resultados. 
Tabla 5.

Resultados sobre la capacidad aeróbica al comparar según PEF.

\begin{tabular}{|c|c|c|c|c|c|}
\hline Estudios & Análisis (HIIT vs MICT) & Intervenciones (n) & Efecto sobre $\mathrm{VO}_{\text {2pico }}(\mathrm{mL} / \mathrm{kg} / \mathrm{min})$ & Valor $p$ & Het. \\
\hline $\begin{array}{l}\text { Gomes-Neto et } \\
\quad \text { al. (2017) }\end{array}$ & $\mathrm{HIIT}^{+}$ & 11 & MD 1.30 & 0.003 & $I^{2}=60 \%$ \\
\hline $\begin{array}{l}\text { Hannan et al. } \\
\qquad(\underline{2018)}\end{array}$ & $\mathrm{HIIT}^{+}$ & 17 & MD 1.15 & $<0.00001$ & $I^{2}=13 \%$ \\
\hline $\begin{array}{l}\text { Pattyn et al. } \\
\qquad \underline{\underline{2018}})\end{array}$ & $\mathrm{HIIT}^{+}$ & 11 & MD 1.40 & 0.0001 & $I^{2}=65 \%$ \\
\hline $\begin{array}{c}\text { Elliott et al. } \\
\qquad \underline{(2015})\end{array}$ & $\mathrm{HIIT}^{+}$ & 6 & WMD 1.53 & 0.0001 & $\mathrm{Chi}^{2}=2.69$ \\
\hline $\begin{array}{l}\text { Pattyn et al. } \\
(2014)\end{array}$ & $\mathrm{HIIT}^{+}$ & 9 & MD 1.60 & 0.03 & $I^{2}=83 \%$ \\
\hline
\end{tabular}

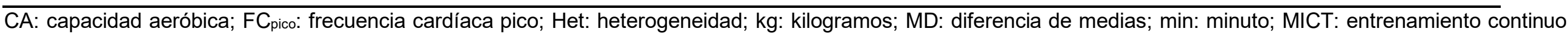
de moderada intensidad; $\mathrm{mL}$ : mililitros; $\mathrm{n}$ : cantidad; $\mathrm{VO}_{2 p i c o}$ : consumo de oxígeno pico; WMD: diferencia de medias ponderada. + Mejoras en comparación al otro PEF. 
En cuanto a los efectos de las características del entrenamiento sobre la capacidad aeróbica, se establecen los resultados en la tabla 6. Dos estudios de alta calidad analizaron los efectos de las siguientes características del HIIT: duración del PEF, duración del intervalo, intensidad y tipo de ejercicio utilizado (Hannan et al., 2018; Pattyn et al., 2018). Programas de intervención de HIIT entre 7 y 12 semanas obtuvieron una mayor mejoría sobre la capacidad aeróbica que programas de intervención $<7$ semanas y $>12$ semanas (Hannan et al., 2018). Programas de intervención de HIIT <12 semanas presentaron una mayor mejoría sobre la capacidad aeróbica que programas de intervención >12 semanas (Pattyn et al., 2018). Programas de intervención de HIIT con una duración del intervalo entre 1 y 3 minutos mostraron una mayor mejoría sobre la capacidad aeróbica que programas de intervención con una duración del intervalo $<1$ minuto y $>3$ minutos (Pattyn et al., 2018). Programas de intervención de HIIT con una intensidad muy alta ( $>90 \% \mathrm{FC}_{\text {pico }}$ $>80 \% \mathrm{VO}_{2 \text { pico }}$ ) del intervalo obtuvieron una mayor mejoría sobre la capacidad aeróbica en comparación programas de intervención con una intensidad alta $\left(<89 \% \mathrm{FC}_{\text {pico }}\right.$ $<79 \% \mathrm{VO}_{2 \text { pico }}$ ) del intervalo (Pattyn et al., 2018). Programas de intervención de HIIT realizados en cinta de correr presentaron una mayor mejoría sobre la capacidad aeróbica en comparación a programas de intervención realizados en cicloergómetro (Pattyn et al., 2018). No se encontraron mejoras significativas en ninguno de estos análisis, sin poder llegar a resultados consistentes. Además, se debe tener cautela con los efectos de las características del HIIT reportados por Pattyn et al. (2018), debido a que el análisis incluye estudios que evaluaron pacientes con EAC y estudios que evaluaron pacientes con insuficiencia cardíaca. 
Tabla 6.

Efectos de las características del HIIT sobre la capacidad aeróbica en los estudios incluidos.

\begin{tabular}{|c|c|c|c|c|c|c|}
\hline Estudios & Características del HIIT & $\begin{array}{c}\text { Análisis } \\
\text { (caracteristicas HIIT vs MICT) }\end{array}$ & $\begin{array}{l}\text { Intervenciones } \\
\text { (n) }\end{array}$ & $\begin{array}{l}\text { Efecto sobre } \mathrm{VO}_{2 \text { pico }} \\
(\mathrm{mL} / \mathrm{kg} / \mathrm{min})\end{array}$ & $\begin{array}{l}\text { Diferencias subgrupos } \\
\text { (valor } p)\end{array}$ & $\begin{array}{l}1^{2} \\
(\%)\end{array}$ \\
\hline \multirow{3}{*}{ Hannan et al. ( $\underline{\text { 2018) }}$} & \multirow{3}{*}{ Duración programa } & $<7$ semanas & 4 & SMD 0.19 & \multirow{3}{*}{0.47} & \multirow{3}{*}{0} \\
\hline & & 7-12 semanas & 11 & SMD 0.43 & & \\
\hline & & $>12$ semanas & 6 & SMD 0.32 & & \\
\hline \multirow[t]{2}{*}{ Pattyn et al. (2018) } & \multirow[t]{2}{*}{ Duración programa } & $<12$ sem & 8 & MD 1.56 & \multirow{2}{*}{0.73} & \multirow{2}{*}{0} \\
\hline & & $>12$ sem & 16 & MD 1.32 & & \\
\hline \multirow{3}{*}{ Pattyn et al. (2018) } & \multirow{3}{*}{ Duración intervalos Al } & $<1$ minuto & 8 & MD 1.27 & \multirow{3}{*}{0.71} & \multirow{3}{*}{0} \\
\hline & & $1-3$ minutos & 5 & MD 2.06 & & \\
\hline & & $>3$ minutos & 11 & MD 1.40 & & \\
\hline \multirow[t]{2}{*}{ Pattyn et al. (2018) } & \multirow{2}{*}{ Intensidad } & muy alta & 15 & MD 1.52 & \multirow{2}{*}{0.55} & \multirow{2}{*}{0} \\
\hline & & alta & 8 & MD 1.06 & & \\
\hline \multirow[t]{2}{*}{ 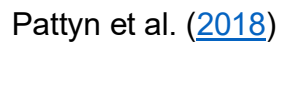 } & \multirow[t]{2}{*}{ Tipo de ejercicio } & cinta de correr & 12 & MD 2.04 & \multirow{2}{*}{0.11} & \multirow{2}{*}{59.9} \\
\hline & & cicloergómetro & 9 & MD 0.99 & & \\
\hline
\end{tabular}

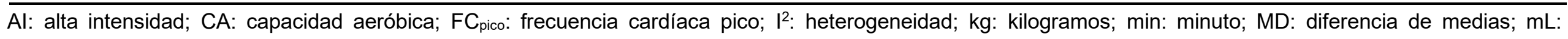
mililitros; $\mathrm{n}$ : cantidad; SMD: diferencia de medias estandarizada; $\mathrm{VO}_{2 p i c o}$ : consumo de oxígeno pico. 


\subsection{Efectos del HIIT vs MICT sobre variables secundarias}

En un estudio de alta calidad se encontraron mejoras significativas del HIIT sobre frecuencia cardíaca pico (+5.11 latidos/minuto) en comparación al MICT (Pattyn et al., 2018). A su vez, se reportaron mejoras significativas del MICT en comparación al HIIT sobre frecuencia cardíaca de reposo (-1.80 latidos/minuto) en 1 estudio de moderada calidad, y sobre peso corporal $(-0.48 / 0.78 \mathrm{~kg})$ en 2 estudios de moderada (Liou et al., 2016) y baja calidad (Pattyn et al., 2014) (ver Tabla 7).

Además, no se encontraron diferencias significativas al comparar los efectos del HIIT y MICT sobre las siguientes variables: calidad de vida (Gomes-Neto et al., 2017; Pattyn et al., 2018), dilatación mediada por flujo, fracción de eyección del ventrículo izquierdo, frecuencia cardíaca de recuperación, nivel de glucosa (Pattyn et al., 2018), nivel de lípidos (Elliott et al., 2015; Pattyn et al., 2018), pendiente de eficiencia de consumo de oxígeno (Pattyn et al., 2018), pendiente de eficiencia ventilatoria (Pattyn et al., 2018, 2014), peso corporal (Pattyn et al.,_2018), presión arterial (Elliott et al., 2015; Pattyn et al., 2018), primer umbral ventilatorio (Pattyn et al., 2018, 2014) y pulso de oxígeno (Pattyn et al., 2018). En todas estas variables el HIIT presentó mejoras no significativas en comparación al MICT(Elliott et al., 2015; Gomes-Neto et al., 2017; Pattyn et al., 2018, 2014), a excepción de la fracción de eyección del ventrículo izquierdo (Pattyn et al., 2018), nivel de lípidos en 1 estudio (Pattyn et al., 2018) y presión arterial en 1 estudio (Elliott et al., 2015) (ver Apéndice 2). 
Tabla 7.

Efectos significativos sobre variables secundarias al comparar según PEF.

\begin{tabular}{|c|c|c|c|c|c|c|}
\hline Estudios & Análisis $_{\mathrm{MICT})}$ (HIIT vs & Variable & $\begin{array}{l}\text { Intervenciones } \\
\text { (n) }\end{array}$ & Efecto (MD) & Valor $p$ & $\begin{array}{l}\mathrm{l}^{2} \\
(\%)\end{array}$ \\
\hline $\begin{array}{c}\text { Pattyn et al. } \\
\qquad \underline{(2018)}\end{array}$ & $\mathrm{HIIT}^{+}$ & $\mathrm{FC}_{\text {pico }}$ & 10 & 5.11 lat $/ \mathrm{min}$ & 0.002 & 0 \\
\hline \multirow{2}{*}{$\begin{array}{l}\text { Liou et al. } \\
\quad(\underline{2016})\end{array}$} & $\mathrm{MICT}^{+}$ & FC reposo & 6 & $-1.80 \mathrm{lat} / \mathrm{min}$ & 0.001 & 0 \\
\hline & & Peso corporal & 7 & $-0.48 \mathrm{~kg}$ & 0.004 & 0 \\
\hline $\begin{array}{l}\text { Pattyn et al. } \\
(\underline{2014})\end{array}$ & $\mathrm{MICT}^{+}$ & Peso corporal & 5 & $-0.78 \mathrm{~kg}$ & 0.05 & 0 \\
\hline
\end{tabular}

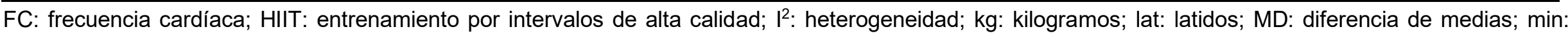
minuto; MICT: entrenamiento continuo de moderada intensidad; $\mathrm{mL}$ : mililitros; $\mathrm{n}$ : cantidad; $\mathrm{VO}_{2 p i c o}$ : consumo de oxígeno pico. + Mejoras en comparación al otro PEF. 


\section{DISCUSIÓN}

El objetivo de esta revisión fue analizar los efectos del HIIT en comparación al MICT sobre la capacidad aeróbica y otras variables relevantes de la RC en pacientes con EAC.

\subsection{Efectos del HIIT vs MICT sobre la capacidad aeróbica}

El HIIT reportó mayores beneficios en comparación al MICT sobre la capacidad aeróbica de pacientes adultos con EAC, con un rango medio de mejoras que osciló entre 1.15-1.78 mL/kg/min del VO $\mathrm{V}_{2 \text { ico }}$ (Elliott et al., 2015; Gomes-Neto et al., 2017; Hannan et al., 2018; Liou et al., 2016; Pattyn et al., 2018, 2014). La magnitud de esta diferencia entre entrenamientos resulta clínicamente relevante ya que por cada 1 $\mathrm{mL} / \mathrm{kg} / \mathrm{min}$ de incremento del $\mathrm{VO}_{2 \text { pico }}$ se reduce aproximadamente un $15 \%$ del riesgo de mortalidad por toda causa y mortalidad cardiovascular en esta población (Pattyn et al., 2014). Estudios anteriores también han encontrado resultados beneficiosos del HIIT sobre la capacidad aeróbica en esta población (Guiraud et al., 2012; Xie, Yan, Cai, y Li, 2017). Los mecanismos potenciales para mayores mejorías incluyen un aumento en el coactivador 1-alfa del receptor activado gamma del proliferador de peroxisoma (PGC-1 $\alpha$ ), mejorando la función mitocondrial y aumentando la tasa máxima de re-captación de Ca2+ en el retículo sarcoplásmico (Wisløff et al., 2007).

En cuanto a las características del entrenamiento, continua siendo difícil establecer un consenso del HIIT debido a la diferencia de protocolos utilizados y al poco análisis de los diferentes elementos del PEF (Cornish et al., 2011; Ribeiro, Boidin, Juneau, Nigam, y Gayda, 2017). En esta revisión se encontró que programas de intervención de HIIT con intensidades muy altas ( $>90 \% \mathrm{FC}_{\text {pico }}->80 \% \mathrm{VO}_{2 \text { pico }}$ ) reportaron mayores mejorías sobre la capacidad aeróbica en comparación a intensidades altas ( $<89 \% \mathrm{FC}_{\text {pico }}-<79 \%$ $\mathrm{VO}_{2 \text { pico }}$ (Pattyn et al., 2018). En la misma línea, estudios anteriores han encontrado que programas de intervención de HIIT con intensidades muy altas ( $>92 \% \mathrm{FC}_{\text {pico }}$ ) generaron mejoras de hasta $2 \mathrm{~mL} / \mathrm{kg} / \mathrm{min}$ del $\mathrm{VO}_{2 \text { pico }}$ en comparación a programas de intervención con intensidades altas (<89\% $\mathrm{FC}_{\text {pico }}$ ) (Moholdt et al., 2014). La intensidad de los intervalos parece ser una de las características del HIIT más importantes para su efectividad sobre la capacidad aeróbica de esta población (Wisløff et al., 2007). Según diferentes estudios, es el parámetro del HIIT que produce mayores adaptaciones en el sistema cardiovascular, como un aumento en el área de corte transversal muscular o una mayor sincronización de las unidades motoras (Smart, Dieberg, y Giallauria, 2013). 
A su vez, programas de intervención de HIIT con menor duración (7-12 semanas) obtuvieron mejoras sobre la capacidad aeróbica en comparación a programas de intervención con mayor duración (>12 semanas) (Hannan et al., 2018; Pattyn et al., 2018). Este resultado puede deberse a una carga de trabajo no incremental luego de las 12 semanas, sin establecerse el principio de progresión del entrenamiento en programas de intervención de HIIT con una duración prolongada (Ballesta García et al., 2019). Sin variación de estímulos y aumento de las cargas del entrenamiento, no se establecen nuevas adaptaciones y mejoras fisiológicas en el organismo del paciente (Taylor et al., 2019). Estudios anteriores establecen que la duración mínima del HIIT para producir adaptaciones significativas sobre la capacidad aeróbica es de 6 semanas, llegando a su mayor efectividad entre las 6 y 12 semanas (Ballesta García et al., 2019; Conraads et al., 2015). Los estudios incluidos en esta revisión analizaron mayor cantidad de intervenciones de HIIT entre 4 y 12 semanas de duración. Por lo tanto, son necesarios más estudios que analicen programas de intervención de HIIT mayores a 12 semanas que atiendan a los principios generales del entrenamiento.

Si bien se desconoce la dosis óptima del HIIT para pacientes cardíacos, la mayoría de la evidencia demuestra mejoras sobre la capacidad aeróbica a través de intervalos de alta intensidad de larga duración (Taylor et al., 2019). Sólo 1 estudio incluido analizó este elemento reportando una mayor mejoría para intervalos de corta duración (1-3 minutos) en comparación a intervalos de menor (<1 minuto) y mayor duración (>3 minutos) (Pattyn et al., 2018). Estudios anteriores no han encontrado diferencias significativas sobre la capacidad aeróbica en esta población al comparar según intervalos de corta y larga duración (Ballesta García et al., 2019). Sin embargo, investigaciones anteriores con intervalos de corta duración (1 minuto) y un volumen de trabajo por sesión menor a 15 minutos no presentaron mejoras sobre la capacidad aeróbica en comparación al MICT (Taylor et al., 2019). A su vez, estudios anteriores establecen que intervalos de alta intensidad de larga duración (3-4 minutos) generaron menor adherencia de los pacientes al HIIT (Ballesta García et al.,_2019). La adherencia al entrenamiento es un elemento importante en la efectividad del PEF (Kraal et al., 2017). En efecto, algunas investigaciones sostienen que los pacientes cardíacos que participan del HIIT prefieren una duración corta del intervalo de alta intensidad, favoreciendo una mayor adherencia al entrenamiento y una mayor mejoría sobre la capacidad aeróbica (Guiraud et al., 2010, 2012). Por lo tanto, la duración del intervalo de alta intensidad debe tener en cuenta las características de los pacientes, el volumen de trabajo a alta intensidad y la proporción trabajo/recuperación. 
La realización del HIIT en cinta de correr presentó mayores mejorías sobre la capacidad aeróbica en comparación al cicloergómetro (Pattyn et al., 2018). El tipo de ejercicio físico utilizado puede asociarse con la cantidad de músculos involucrados en la actividad o con la preferencia y adherencia del paciente al HIIT, siendo un elemento importante a tener en cuenta en los efectos del PEF (Kraal et al., 2017).

Para todas estas características del HIIT analizadas en los estudios incluidos, no se pueden establecer conclusiones consistentes debido a que no se hallaron resultados significativos. Por lo tanto, son necesarios más estudios que comparen diferentes protocolos del HIIT para establecer la prescripción más eficiente en la RC de pacientes con EAC. La prescripción del HIIT puede tener muchas variantes que modifiquen los resultados sobre la capacidad aeróbica en estos pacientes: intensidad del período de recuperación, duración de los intervalos o proporción trabajo/recuperación, entre otros (Ballesta García et al., 2019; Taylor et al., 2019). Además, es imprescindible la supervisión y prescripción del HIIT de forma individual según las características clínicas y personales de cada paciente para una prevención secundaria más efectiva en esta población (Cornish et al., 2011).

\subsection{Efectos del HIIT vs MICT sobre variables secundarias}

El HIIT se asoció a mejoras significativas sobre frecuencia cardíaca pico en comparación al MICT (Pattyn et al., 2018). La frecuencia cardíaca pico es un parámetro cardio-respiratorio importante en el pronóstico de la EAC (Pattyn et al., 2018). Mayor intensidad del ejercicio a través del HIIT provocaría mayor trabajo del músculo cardíaco para proporcionar oxígeno a los músculos que están en movimiento, resultando en mejoras sobre el volumen sistólico y la frecuencia cardíaca pico (Pattyn et al., 2018).

Mientras que el MICT se asoció a mejoras significativas sobre frecuencia cardíaca de reposo y peso corporal en comparación al HIIT (Liou et al., 2016; Pattyn et al., 2014). Tanto la frecuencia cardíaca de reposo como el peso corporal son factores de riesgo cardiovasculares importantes en esta población (Fox et al., 2007; Medina-Inojosa et al.,2018). Las mejoras del MICT sobre frecuencia cardíaca de reposo y peso corporal podrían estar asociadas a un mayor gasto calórico, obteniéndose a través de un mayor volumen del MICT en comparación al HIIT (Vanhees et al., 2012). Sin embargo, los estudios que encontraron mejoras del MICT sobre la frecuencia cardíaca de reposo y el peso corporal presentaron una calidad metodológica moderada (Liou et al., 2016) y baja (Pattyn et al., 2014). Además, 1 estudio de alta calidad incluido en esta revisión 
encontró mejoras no significativas del HIIT en comparación al MICT sobre estas variables (Pattyn et al., 2018). Por lo tanto, son necesarios más estudios con una correcta metodología para llegar a conclusiones consistentes sobre estas y otras variables relevantes en la $\mathrm{RC}$ de pacientes con EAC.

\subsection{Seguridad HIIT}

A pesar de que el HIIT con una alta intensidad de trabajo resultó beneficioso sobre la capacidad aeróbica, también puede conducir a un aumento en el riesgo de eventos cardíacos adversos (P. D. Thompson et al., 2007; P. Thompson y Williams, 2014). El HIIT debe realizarse con aprobación médica, en pacientes estables, con la supervisión de un profesional del ejercicio y con una progresión adecuada (Ribeiro et al., 2017). Rongmo y colegas encontraron un mayor riesgo en personas que realizaron HIIT en comparación a personas que realizaron MICT (i.e. 1 evento / 23 horas vs 1 evento / 129 horas, respectivamente) (Rognmo et al., 2012). Sin embargo, establecieron un cálculo de potencia que muestra que para un ensayo aleatorizado con potencia adecuada se requieren 20.500 pacientes y 750.000 horas de entrenamiento (Rognmo et al., 2012). Por lo tanto, sigue siendo difícil sacar conclusiones sobre la relación riesgo/seguridad del HIIT en estos pacientes.

\subsection{Principales limitaciones}

Como principal limitante y riesgo de sesgo encontramos la búsqueda de estudios publicados en dos idiomas y en una sola base de datos, proporcionando un número reducido de artículos que cumplieron los criterios de inclusión. Una limitación significativa de esta revisión es la incapacidad de agrupar los resultados y calcular los efectos según diferentes características del entrenamiento que no fueron analizadas en los estudios. Además, esta revisión es limitada porque se centró solo en dos tipos de programas de entrenamiento, sin incluirse en el análisis aspectos como el tratamiento farmacológico o etapa de la enfermedad. En cuanto a la información que presentan las revisiones sistemáticas y meta-análisis incluidos, se encuentra una gran variedad en los parámetros de los protocolos de los entrenamientos y heterogeneidad alta en los resultados, por lo que las conclusiones que se puedan sacar a partir de este tipo de investigaciones en estos apartados deben ser tenidas con cautela. 


\section{CONCLUSIONES}

El HIIT presentó mejoras significativas en comparación al MICT sobre la capacidad aeróbica y la frecuencia cardíaca pico en pacientes con EAC. Según esta revisión, programas de intervención de HIIT con una duración entre 7-12 semanas, realizados en cinta de correr y con intervalos de muy alta intensidad (>90\% $\mathrm{FC}_{\text {pico }}->80 \% \mathrm{VO}_{2 \text { pico }}$ ) entre 1-3 minutos, resultaron los parámetros más adecuados para mejorar la capacidad aeróbica en esta patología. Sin embargo, los efectos de estos parámetros del HIIT no fueron significativos al comparar con otros rangos, siendo necesarios más estudios que confirmen estos resultados. A su vez, son necesarios más estudios que comparen otros elementos del protocolo del HIIT como: programas de corta duración y programas de larga duración con una progresión adecuada; intervalos de alta intensidad de larga duración y corta duración, en relación a la adherencia al entrenamiento; tipo de recuperación activa y pasiva; períodos de recuperación de larga duración y corta duración; diferentes volúmenes de trabajo por sesión; y diferentes proporciones de trabajo/recuperación. Además, son necesarios más estudios con una correcta metodología que analicen los efectos del HIIT en comparación al MICT sobre la frecuencia cardíaca de reposo, el peso corporal, eventos cardíacos adversos y otras variables relevantes de la RC en esta población, que permitan llegar a conclusiones consistentes.

Fondos: No se utilizaron fuentes de financiamiento para ayudar en la preparación de este artículo.

Conflictos de interés: Ninguno. 


\section{REFERENCIAS.}

Se resaltan con un asterisco $\left({ }^{*}\right)$ las referencias que se utilizaron para los análisis del presente artículo, como se menciona en la Tabla 1.

Anderson, L., Oldridge, N., Thompson, D. R., Zwisler, A.-D., Rees, K., Martin, N., y Taylor, R. S. (2016). Exercise-Based Cardiac Rehabilitation for Coronary Heart Disease: Cochrane Systematic Review and Meta-Analysis. Journal of the American College of Cardiology, 67(1), 1-12. doi: https://doi.org/10.1016/j.jacc.2015.10.044

Ballesta García, I., Rubio Arias, J. Á., Ramos Campo, D. J., Martínez González-Moro, I., y Carrasco Poyatos, M. (2019). High-intensity Interval Training Dosage for Heart Failure and Coronary Artery Disease Cardiac Rehabilitation. A Systematic Review and Meta-analysis. Revista Espanola de Cardiologia, 72(3), 233-243. doi: https://doi.org/10.1016/j.rec.2018.02.015

Clark, A. M., Hartling, L., Vandermeer, B., y McAlister, F. A. (2005). Meta-analysis: secondary prevention programs for patients with coronary artery disease. Annals of Internal Medicine, 143(9), 659-672. doi: https://doi.org/10.7326/0003-4819-1439-200511010-00010

Conraads, V. M., Pattyn, N., De Maeyer, C., Beckers, P. J., Coeckelberghs, E., Cornelissen, V. A., ... Vanhees, L. (2015). Aerobic interval training and continuous training equally improve aerobic exercise capacity in patients with coronary artery disease: The SAINTEX-CAD study. International Journal of Cardiology, 179, 203210. doi: https://doi.org/10.1016/J.IJCARD.2014.10.155

Cornish, A. K., Broadbent, S., y Cheema, B. S. (2011). Interval training for patients with coronary artery disease: a systematic review. European Journal of Applied Physiology, 111(4), 579-589. doi: https://doi.org/10.1007/s00421-010-1682-5

*Elliott, A. D., Rajopadhyaya, K., Bentley, D. J., Beltrame, J. F., y Aromataris, E. C. (2015). Interval training versus continuous exercise in patients with coronary artery disease: a meta-analysis. Heart, Lung \& Circulation, 24(2), 149-157. doi: https://doi.org/10.1016/j.hlc.2014.09.001

Fox, K., Borer, J. S., Camm, A. J., Danchin, N., Ferrari, R., Lopez Sendon, J. L., ... Heart Rate Working Group. (2007). Resting Heart Rate in Cardiovascular Disease. Journal of the American College of Cardiology, 50(9), 823-830. doi: https://doi.org/10.1016/j.jacc.2007.04.079

*Gomes-Neto, M., Duraes, A. R., dos Reis, H. F., Neves, V. R., Martinez, B. P., y Carvalho, V. O. (2017). High-intensity interval training versus moderate-intensity continuous training on exercise capacity and quality of life in patients with coronary artery disease: A systematic review and meta-analysis. European Journal of Preventive Cardiology, 24(16), 1696-1707. doi: https://doi.org/10.1177/2047487317728370

Guiraud, T., Juneau, M., Nigam, A., Gayda, M., Meyer, P., Mekary, S., ... Bosquet, L. (2010). Optimization of high intensity interval exercise in coronary heart disease. European Journal of Applied Physiology, 108(4), 733-740. doi: 
https://doi.org/10.1007/s00421-009-1287-z

Guiraud, T., Nigam, A., Gremeaux, V., Meyer, P., Juneau, M., y Bosquet, L. (2012). High-intensity interval training in cardiac rehabilitation. Sports Medicine (Auckland, N.Z.), 42(7), 587-605. doi: https://doi.org/10.2165/11631910-000000000-00000

*Hannan, A. L., Hing, W., Simas, V., Climstein, M., Coombes, J. S., Jayasinghe, R., ... Furness, J. (2018). High-intensity interval training versus moderateintensity continuous training within cardiac rehabilitation: a systematic review and metaanalysis. Open Access Journal of Sports Medicine, 9, 1-17. doi: https://doi.org/10.2147/OAJSM.S150596

Kodama, S., Saito, K., Tanaka, S., Maki, M., Yachi, Y., Asumi, M., ... Sone, H. (2009). Cardiorespiratory fitness as a quantitative predictor of all-cause mortality and cardiovascular events in healthy men and women: a meta-analysis. JAMA, 301(19), 2024-2035. doi: https://doi.org/10.1001/jama.2009.681

Kraal, J. J., Vromen, T., Spee, R., Kemps, H. M. C., y Peek, N. (2017). The influence of training characteristics on the effect of exercise training in patients with coronary artery disease: Systematic review and meta-regression analysis. International Journal of Cardiology, 245, 52-58. doi: https://doi.org/10.1016/j.jjcard.2017.07.051

*Liou, K., Ho, S., Fildes, J., y Ooi, S.-Y. (2016). High Intensity Interval versus Moderate Intensity Continuous Training in Patients with Coronary Artery Disease: A Metaanalysis of Physiological and Clinical Parameters. Heart, Lung y Circulation, 25(2), 166-174. doi: https://doi.org/10.1016/j.hlc.2015.06.828

Medina-Inojosa, J. R., Somers, V. K., Thomas, R. J., Jean, N., Jenkins, S. M., Gomez-Ibarra, M. A., ... Lopez-Jimenez, F. (2018). Association Between Adiposity and Lean Mass With Long-Term Cardiovascular Events in Patients With Coronary Artery Disease: No Paradox. Journal of the American Heart Association, 7(10). doi: https://doi.org/10.1161/JAHA.117.007505

Mezzani, A., Hamm, L. F., Jones, A. M., McBride, P. E., Moholdt, T., Stone, J. A., ... Canadian Association of Cardiac Rehabilitation. (2013). Aerobic exercise intensity assessment and prescription in cardiac rehabilitation: a joint position statement of the European Association for Cardiovascular Prevention and Rehabilitation, the American Association of Cardiovascular and Pulmonary Rehabilitat. European Journal of Preventive Cardiology, 20(3), 442-467. doi: https://doi.org/10.1177/2047487312460484

Moher, D., Liberati, A., Tetzlaff, J., Altman, D. G., y Group, T. P. (2009). Preferred Reporting Items for Systematic Reviews and Meta-Analyses: The PRISMA Statement. PLoS Medicine, 6(7), e1000097. doi: https://doi.org/10.1371/journal.pmed.1000097

Moholdt, T., Madssen, E., Rognmo, Ø., y Aamot, I. L. (2014). The higher the better? Interval training intensity in coronary heart disease. Journal of Science and Medicine in Sport, 17(5), 506-510. doi: https://doi.org/10.1016/j.jsams.2013.07.007

Montalescot, G., Sechtem, U., Achenbach, S., Andreotti, F., Arden, C., Budaj, A., ... Yildirir, A. (2013). 2013 ESC guidelines on the management of stable coronary artery disease. European Heart Journal, 34(38), 2949-3003. doi: 
https://doi.org/10.1093/eurheartj/eht296

*Pattyn, N., Beulque, R., y Cornelissen, V. (2018). Aerobic Interval vs. Continuous Training in Patients with Coronary Artery Disease or Heart Failure: An Updated Systematic Review and Meta-Analysis with a Focus on Secondary Outcomes. Sports Medicine, 48(5), 1189-1205. doi: https://doi.org/10.1007/s40279-018-08855

*Pattyn, N., Coeckelberghs, E., Buys, R., Cornelissen, V. A., y Vanhees, L. (2014). Aerobic Interval Training vs. Moderate Continuous Training in Coronary Artery Disease Patients: A Systematic Review and Meta-Analysis. Sports Medicine, 44(5), 687-700. doi: https://doi.org/10.1007/s40279-014-0158-x

Piepoli, M. F., Ugo, C., Carré, F., Heuschmann, P., Hoffmann, U., Verschuren, M., ... Schmid, J.-P. (2010). Secondary prevention through cardiac rehabilitation: physical activity counselling and exercise training: key components of the position paper from the Cardiac Rehabilitation Section of the European Association of Cardiovascular Prevention and Rehabilitat. European Heart Journal, 31(16), 1967-1974. doi: https://doi.org/10.1093/eurheartj/ehq236

Ribeiro, P. A. B., Boidin, M., Juneau, M., Nigam, A., y Gayda, M. (2017). High-intensity interval training in patients with coronary heart disease: Prescription models and perspectives. Annals of Physical and Rehabilitation Medicine, 60(1), 50-57. doi: https://doi.org/10.1016/j.rehab.2016.04.004

Rognmo, Ø., Hetland, E., Helgerud, J., Hoff, J., y Slørdahl, S. A. (2004). High intensity aerobic interval exercise is superior to moderate intensity exercise for increasing aerobic capacity in patients with coronary artery disease. European Journal of Cardiovascular Prevention and Rehabilitation: Official Journal of the European Society of Cardiology, Working Groups on Epidemiology \& Prevention and Cardiac Rehabilitation and Exercise Physiology, 11(3), 216-222. doi: https://doi.org/10.1097/01.hjr.0000131677.96762.0c

Rognmo, Ø., Moholdt, T., Bakken, H., Hole, T., Mølstad, P., Myhr, N. E., ... Wisløff, U. (2012). Cardiovascular risk of high- versus moderate-intensity aerobic exercise in coronary heart disease patients. Circulation, 126(12), 1436-1440. doi: https://doi.org/10.1161/CIRCULATIONAHA.112.123117

Shea, B., Reeves, B. C., Wells, G., Thuku, M., Hamel, C., Moran, J., ... Henry, D. A. (2017a). AMSTAR 2: a critical appraisal tool for systematic reviews that include randomised or non-randomised studies of healthcare interventions, or both. BMJ, 1-9. doi: https://doi.org/10.1136/bmj.j4008

Shea, B., Reeves, B., Wells, G., Thuku, M., Hamel, C., Moran, J., ... Henry, D. (2017b). AMSTAR Checklist.

Smart, N. A., Dieberg, G., y Giallauria, F. (2013). Intermittent versus continuous exercise training in chronic heart failure: A meta-analysis. International Journal of Cardiology, 166(2), 352-358. doi: https://doi.org/10.1016/j.ijcard.2011.10.075

Taylor, J. L., Holland, D. J., Spathis, J. G., Beetham, K. S., Wisløff, U., Keating, S. E., y Coombes, J. S. (2019). Guidelines for the delivery and monitoring of high intensity interval training in clinical populations. Progress in Cardiovascular Diseases, 62(2), 140-146. doi: https://doi.org/10.1016/j.pcad.2019.01.004 
Thompson, P. D., Franklin, B. A., Balady, G. J., Blair, S. N., Corrado, D., Estes, N. A. M., ... American College of Sports Medicine. (2007). Exercise and acute cardiovascular events placing the risks into perspective: a scientific statement from the American Heart Association Council on Nutrition, Physical Activity, and Metabolism and the Council on Clinical Cardiology. Circulation, 115(17), 23582368. doi: https://doi.org/10.1161/CIRCULATIONAHA.107.181485

Thompson, P., y Williams, P. (2014). Increased cardiovascular disease mortality associated with excessive exercise in heart attack survivors. Mayo Clinic Proceedings, 89(9), 1187-1194. doi: https://doi.org/10.1016/j.mayocp.2014.05.006

Valkeinen, H., Aaltonen, S., y Kujala, U. M. (2010). Effects of exercise training on oxygen uptake in coronary heart disease: a systematic review and meta-analysis. Scandinavian Journal of Medicine \& Science in Sports, 20(4), 545-555. doi: https://doi.org/10.1111/j.1600-0838.2010.01133.x

Vanhees, L., Geladas, N., Hansen, D., Kouidi, E., Niebauer, J., Reiner, Ž., ... Vanuzzo, D. (2012). Importance of characteristics and modalities of physical activity and exercise in the management of cardiovascular health in individuals with cardiovascular risk factors: recommendations from the EACPR (Part II). European Journal of Preventive Cardiology, 19(5), 1005-1033. doi: https://doi.org/10.1177/1741826711430926

Wisløff, U., Støylen, A., Loennechen, J. P., Bruvold, M., Rognmo, Ø., Haram, P. M., ... Skjærpe, T. (2007). Superior Cardiovascular Effect of Aerobic Interval Training Versus Moderate Continuous Training in Heart Failure Patients. Circulation, 115(24), 3086-3094. doi: https://doi.org/10.1161/CIRCULATIONAHA.106.675041

Woodruffe, S., Neubeck, L., Clark, R. A., Gray, K., Ferry, C., Finan, J., ... Briffa, T. G. (2015). Australian Cardiovascular Health and Rehabilitation Association (ACRA) core components of cardiovascular disease secondary prevention and cardiac rehabilitation 2014. Heart, Lung \& Circulation, 24(5), 430-441. doi: https://doi.org/10.1016/j.hlc.2014.12.008

World Health Organization [WHO] (2011). Global Atlas on cardiovascular disease prevention and control. Geneva: World Health Organization, World Heart Federation, World Stroke Organization. Retrieved from https://www.who.int/cardiovascular_diseases/publications/atlas_cvd/en/

World Health Organization [WHO]. (2016a). Cardiovascular diseases 2016. Retrieved April 1, 2019, from https://www.who.int/es/news-room/factsheets/detail/cardiovascular-diseases-(cvds)

World Health Organization [WHO]. (2016b). WHO | Top 10 causes of death 2016. Retrieved April 1, 2019, from https://www.who.int/news-room/factsheets/detail/the-top-10-causes-of-death

Xie, B., Yan, X., Cai, X., y Li, J. (2017). Effects of High-Intensity Interval Training on Aerobic Capacity in Cardiac Patients: A Systematic Review with Meta-Analysis. BioMed Research International, 2017, 1-16. doi: https://doi.org/10.1155/2017/5420840 


\section{MATERIAL SUPLEMENTARIO}

7.1. Apéndice 1: Lista de las 24 distintas intervenciones incluidas en las 6 revisiones sistemáticas y meta-análisis analizados en el presente manuscrito

Amundsen, B.H., Rognmo, $\varnothing$, Hatlen-Rebhan, G., y Slørdahl, S.A. (2008). Highintensity aerobic exercise improves diastolic function in coronary artery disease. Scand Cardiovasc J, 42(2), 110-117.

Benetti, M, Araujo, C.L., y Santos, R.Z. (2010). Cardiorespiratory fitness and quality of life at different exercise intensities after myocardial infarction. Arq Bras Cardiol, 95(3), 399-404.

Cardozo, G.G., Oliveira, R.B., y Farinatti, P.T. (2015). Effects of high intensity interval versus moderate continuous training on markers of ventilatory and cardiac efficiency in coronary heart disease patients. ScientificWorld-Journal.

Conraads, V.M., Pattyn, N, De Maeyer, C, Beckers, P.J., Coeckelberghs, E., Cornelissen, V.A., ... Denollet, J. (2015). Aerobic interval training and continuous training equally improve aerobic exercise capacity in patients with coronary artery disease: the SAINTEX-CAD study. Int J Cardiol, 179, 203-210.

Currie, K.D., Bailey, K.J., Jung, M.E., McKelvie, R.S., y MacDonald, M.J. (2015). Effects of resistance training combined with moderate-intensity endurance or lowvolume high-intensity interval exercise on cardiovascular risk factors in patients with coronary artery disease. J Sci Med Sport, 18(6), 637-642.

Currie, K.D., Dubberley, J.B., McKelvie, R.S., y MacDonald, M.J. (2013). Low-volume, high-intensity interval training in patients with CAD. Med Sci Sports Exerc, 45(8), 1436-1442.

Dimopoulos, S., Anastatiou-Nana, M., Sakellariou, D., Drakos, S., Kapsimalakou, S., Maroulidis, G., ... Roditis, P. (2006). Effects of exercise rehabilitation program on heart rate recovery in patients with chronic heart failure. Eur $J$ Cardiovasc Prev Rehabil, 13(1), 67-73.

lellamo, F., Manzi V., Caminiti, G., Vitale, C., Castagna, Cet., Massaro, M., ... Franchini, A. (2013) Matched dose interval and continuous exercise training induce similar cardiorespiratory and metabolic adaptations in patients with heart failure. Int J Cardiol, 167(6), 2561-5.

Jaureguizar, K.V., Vicente-Campos, D., Bautista, L.R., de la Peña, C.H., Gómez, M., y Rueda, M.J., Fernández, I. (2016). Effect of highintensity interval versus continuous exercise training on functional capacity and quality of life in patients with coronary artery disease: a randomized clinical trial. J Cardiopulm Rehabil Prev, 36(2), 96-105. 
Jensen, B.E., Fletcher, B.J., Rupp, J.C., Fletcher, G.F., Lee, J,Y., y Oberman, A. (1996). Training level comparison study. Effect of high and low intensity exercise on ventilatory threshold in men with coronary artery disease. $J$ Cardiopulm Rehabil, 16(4), 227-232.

Keteyian, S.J., Hibner, B.A., Bronsteen, K., Kerrigan, D., Aldred, H.A., Reasons, L.M., y ... Saval, M.A. (2014). Greater improvement in cardiorespiratory fitness using higher-intensity interval training in the standard cardiac rehabilitation setting. $J$ Cardiopulm Rehabil Prev, 34(2), 98-105.

Kim, C., Choi, H.E., y Lim, M.H. (2015). Effect of high interval training in acute myocardial infarction patients with drug-eluting stent. Am J Phys Med Rehabil, 94 (10 Suppl 1), 879-886.

Madssen, E., Moholdt, T., Videm, V., Wisløff, U., Hegbom, y K., Wiseth, R. (2014). Coronary atheroma regression and plaque characteristics assessed by grayscale and radiofrequency intravascular ultrasound after aerobic exercise. Am J Cardiol, $114,1504-11$.

Möbius-Winkler, S., Uhlemann, M., Adams, V., Sandri, M., Erbs, S., Lenk, K., ... Mangner, N. (2016). Coronary collateral growth induced by physical exercise: results of the impact of intensive exercise training on coronary collateral circulation in patients with stable coronary artery disease (EXCITE) trial. Circulation, 133(15), 1438-1448.

Moholdt, T., Aamot, IL., Granøien, I., Gjerde, L., Myklebust, G., Walderhaug, L., ... Brattbakk, L. (2012). Aerobic interval training increases peak oxygen uptake more than usual care exercise training in myocardial infarction patients: a randomized controlled study. Clin Rehabil, 26(1), 33-44.

Moholdt, T., Aamot, I.L., Granoien, I., Gjerde, L., Myklebust, G., Walderhaug, L., ... Hole, T. (2011). Long-term follow-up after cardiac rehabilitation: A randomized study of usual care exercise training versus aerobic interval training after myocardial infarction. Int J Cardiol, 152, 388-390.

Moholdt, T.T., Amundsen, B.H., Rustad, L.A., Wahba, A., Løvø, K.T., Gullikstad, L.R., ... Bye, A. (2009). Aerobic interval training versus continuous moderate exercise after coronary artery bypass surgery: a randomized study of cardiovascular effects and quality of life. Am Heart J, 158(6), 1031-1037.

Pattyn, N., Vanhees, L., Cornelissen, V.A., Coeckelberghs, E., De Maeyer, C., Goetschalckx, K., ... Possemiers, N. (2016). The longterm effects of a randomized trial comparing aerobic interval versus continuous training in coronary artery disease patients: 1 -Year data from the SAINTEX-CAD study. Eur J Prev Cardiol, 23, 1154-1164.

Prado, D.M., Rocco, E.A., Silva, A.G., Rocco, D.F., Pacheco, M.T., Silva, P.F., y Furlan, V. (2016). Effects of continuous vs interval exercise training on oxygen 
uptake efficiency slope in patients with coronary artery disease. Braz J Med Biol Res, 49(2), e4890.

Rocco, E.A., Prado, D.M., Silva, A.G., Lazzari, J.M., Bortz, P.C., Rocco, D.F., .. Rosa, C.G. (2012). Effect of continuous and interval exercise training on the PETCO2 response during a graded exercise test in patients with coronary artery disease. Clinics (Sao Paulo), 67(6), 623-628.

Rognmo, Ø., Hetland, E., Helgerud, J., Hoff, J., y Slørdahl, S.A. (2004). High intensity aerobic interval exercise is superior to moderate intensity exercise for increasing aerobic capacity in patients with coronary artery disease. Eur $J$ Cardiovasc Prev Rehabil, 11(3), 216-222.

Smart, N.A., y Steele, M. A. (2012). A comparison of 16 weeks of continuous vs intermittent exercise training in chronic heart failure patients. Congest Heart Fail, 18(4), 205-11.

Warburton, D.E., McKenzie, D.C., Haykowsky, M.J., Taylor, A., Shoemaker, P., Ignaszewski, A.P., y Chan, S.Y. (2005). Effectiveness of high-intensity interval training for the rehabilitation of patients with coronary artery disease. Am J Cardiol, 95(9), 1080-1084.

Wisløff, U., Støylen, A., Loennechen, J.P., Bruvold, M., Rognmo, Ø., Haram, P.M., ...Tjønna, A.E. (2007). Superior cardiovascular effect of aerobic interval training versus moderate continuous training in heart failure patients: a randomized study. Circulation, 115(24), 3086-94. 
7.2. Apéndice 2 (efectos no significativos del HIIT vs MICT sobre variables secundarias).

\begin{tabular}{|c|c|c|c|c|c|c|c|}
\hline Estudios & Tipo de variable & Variable & $\begin{array}{c}\text { Análisis (HIIT vs } \\
\text { MICT) }\end{array}$ & $\begin{array}{l}\text { Intervenciones } \\
\text { (n) }\end{array}$ & Efecto (MD) & Valor $p$ & $\begin{array}{l}1^{2} \\
(\%)\end{array}$ \\
\hline \multirow[t]{13}{*}{$\begin{array}{c}\text { Pattyn et al. } \\
(\underline{2018)})\end{array}$} & $\begin{array}{l}\text { Parámetros } \\
\text { cardiorespiratorios }\end{array}$ & FC recuperación & $\mathrm{HIIT}^{++}$ & 7 & 0.42 & 0.76 & 50 \\
\hline & & OUES & $\mathrm{HIIT}^{+}$ & 3 & 73.37 & 0.36 & 26 \\
\hline & & puv & $\mathrm{HIIT}^{+}$ & 7 & 0.73 & 0.14 & 44 \\
\hline & & pulso de oxígeno & $\mathrm{HIIT}^{+}$ & 4 & 0.52 & 0.22 & 0 \\
\hline & & $\mathrm{VE} / \mathrm{VCO}_{2}$ & $\mathrm{HIIT}^{+}$ & 5 & 0.50 & 0.27 & 0 \\
\hline & $\begin{array}{l}\text { Factores de riesgo } \\
\text { cardiovascular }\end{array}$ & glucosa & $\mathrm{HIIT}^{+}$ & 3 & 0.06 & 0.69 & 0 \\
\hline & & triglicéridos & $\mathrm{MICT}^{+}$ & 4 & 0.02 & 0.77 & 0 \\
\hline & & colesterol LDL & $\mathrm{MICT}^{+}$ & 4 & 0.12 & 0.28 & 29 \\
\hline & & colesterol HDL & - & 4 & 0 & 0.96 & 0 \\
\hline & & SBP & $\mathrm{HIIT}^{+}$ & 5 & 2.67 & 0.29 & 27 \\
\hline & & DBP & $\mathrm{HIIT}^{+}$ & 5 & 0.27 & 0.48 & 46 \\
\hline & & peso corporal & $\mathrm{HIIT}^{+}$ & 6 & 0.67 & 0.37 & 0 \\
\hline & & FC reposo & $\mathrm{HIIT}^{+}$ & 8 & 1.19 & 0.18 & 0 \\
\hline
\end{tabular}




\begin{tabular}{|c|c|c|c|c|c|c|c|}
\hline Estudios & Tipo de variable & Variable & $\begin{array}{c}\text { Análisis (HIIT vs } \\
\text { MICT) }\end{array}$ & $\begin{array}{l}\text { Intervenciones } \\
\text { (n) }\end{array}$ & Efecto (MD) & Valor $p$ & $\begin{array}{l}1^{2} \\
(\%)\end{array}$ \\
\hline \multirow{2}{*}{$\begin{array}{l}\text { Pattyn et al. } \\
(\underline{2018})\end{array}$} & Función vascular & FMD & $\mathrm{HIIT}^{+}$ & 3 & 0.08 & 0.81 & 0 \\
\hline & Función cardíaca & FEVI & $\mathrm{MICT}^{+}$ & 3 & 0.56 & 0.57 & 0 \\
\hline $\begin{array}{l}\text { Gomes. Neto } \\
\text { et al. }(\underline{2017})\end{array}$ & Calidad de vida & calidad de vida & $\mathrm{HIIT}^{+}$ & 2 & 0.07 & 0.30 & 0 \\
\hline \multirow[t]{2}{*}{$\begin{array}{c}\text { Elliott et al. } \\
(\underline{\underline{2015}})\end{array}$} & $\begin{array}{l}\text { Factores de riesgo } \\
\text { cardiovascular }\end{array}$ & colesterol HDL & $\mathrm{HIIT}^{+}$ & 2 & 0.04 & 0.1 & nse \\
\hline & & SBP & $\mathrm{MICT}^{+}$ & 3 & 3.44 & 0.08 & 10.65 \\
\hline \multirow[t]{2}{*}{$\begin{array}{c}\text { Pattyn et al. } \\
(\underline{2014})\end{array}$} & $\begin{array}{c}\text { Parámetros } \\
\text { cardiorespiratorios }\end{array}$ & $\mathrm{VE} / \mathrm{VCO}_{2}$ & $\mathrm{HIIT}^{+}$ & 5 & 1.06 & 0.19 & 65 \\
\hline & & puv & $\mathrm{HIIT}^{+}$ & 6 & 0.35 & 0.54 & 87 \\
\hline
\end{tabular}

DBP: presión arterial diastólica; FC: frecuencia cardíaca; FEVI: fracción de eyección del ventrículo izquierdo; FMD: dilatación mediada por flujo; HDL: lipoproteínas de alta densidad; HIIT: entrenamiento por intervalos de alta calidad; I $^{2}$ : heterogeneidad; LDL: lipoproteínas de baja densidad; MD: diferencia de medias; MICT: entrenamiento continuo de moderada intensidad; n: cantidad; nse: no se especifica; OUES: pendiente de eficiencia

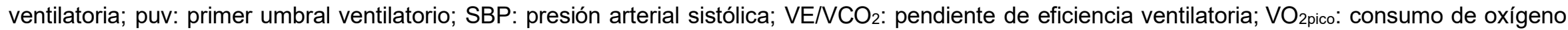
pico. + Mejoras en comparación al otro PEF. 